\title{
RIDAS
}

\section{Aproximación a los impactos y beneficios del aprendizaje servicio en la Universidad de Zaragoza}

\author{
Mạ Isabel Saz Gil sazgil@unizar.es \\ Rosario Marta Ramo Garzarán rmarta@unizar.es \\ Universidad de Zaragoza, España.
}

\section{Resumen}

El aprendizaje servicio es una estrategia didáctica innovadora que posibilita la participación del estudiante en propuestas de intervención social, en las que además de aplicar los conocimientos que adquiere en el espacio educativo convencional, se involucra en determinadas problemáticas con una importante repercusión social, generando sinergias con las entidades sociales. La propuesta que presentamos pretende implicar de forma paulatina a las distintas titulaciones del Campus de Teruel -Magisterio, Enfermería, Bellas Artes, Psicología, Administración y Dirección de Empresas, Ingeniería Informática, Ingeniería Electrónica y Automática-, a través de la implementación de la metodología en diferentes asignaturas. El objetivo es desarrollar un proceso acumulativo de experiencias que permitan tejer redes estables de colaboración y confianza entre el Campus turolense y las entidades del tercer sector de la provincia, poniendo en relación a los estudiantes universitarios con las organizaciones no lucrativas. En esta primera fase se muestra la experiencia realizada en una titulación y la propuesta en otra titulación, ambas en la Facultad de Ciencias Sociales y Humanas.

\section{Palabras clave}

Innovación Social, aprendizaje servicio, organización no lucrativa, responsabilidad social; universidad. 


\title{
An Approach to the Impacts and Benefits of Service-Learning at the University of Zaragoza
}

\begin{abstract}
Service-learning is an innovative teaching strategy that enables student's participation and social intervention, applying their knowledge in issues with social impact and generating synergies with social organizations. Our proposal aims to involve gradually different degrees to Teruel Campus -Education, Nursing, Fine Arts, Psychology, Business Administration, Computer Engineering, and Electrical Engineering- through the implementation of the methodology in different subjects. The aim is to develop a process of experiences to make networks of collaboration and trust between Teruel Campus and third sector organizations in the village. In this first phase is shown the experience in a degree and the proposal in another degree, both in the Faculty of Social and Human Sciences.
\end{abstract}

\section{Palabras clave}

Social Innovation, Service-Learning, Nonprofit Organization, Social Responsibility, University. 


\section{Introducción}

La metodología del saber hacer "aprendiendo haciendo" está teniendo diferentes impactos no solo en la forma de entender la combinación teoría práctica, sino en la forma de plantear desde diferentes instituciones programas y proyectos donde la unión de los distintos sectores aplican su propia estrategia de implementación, adaptando la realidad de las diferentes instituciones a la formación académica y viceversa, por lo que supone una innovación educativa y una innovación en el plano social, un poner en valor buenas prácticas que ya se vienen desarrollando, que no vienen definidas como aprendizaje servicio (ApS), o bien no se han desarrollado a fondo las posibilidades del vínculo entre el aprendizaje curricular y el servicio a la comunidad.

Como señala Arranz (2011), la universidad como tal tiene que aprehender este modo más rico de contemplar la educación superior, sin limitarse a proporcionar el aprendizaje de conocimientos especializados y habilidades técnicas de actuación. La introducción de valores sociales e individuales en la cultura universitaria favorecerá la inserción de los mismos en el currículum de las diferentes titulaciones. Toda la comunidad universitaria tiene el derecho, y también sería deseable que tuviese el deber, de estar informada acerca de la necesaria responsabilidad social, como individuo y como ciudadano.

La estrategia de desarrollo del ApS ha consistido en colocarse en el corazón de las organizaciones ya existentes en cada territorio, estimulando que lo identifiquen como útil para sus fines y que encuentren sentido en unir esfuerzos y coordinarse con otros agentes de su entorno para promoverla más allá de su propio ámbito de actuación (Batlle, 2010). La universidad retoma esta propuesta y referente y dentro de lo que podríamos llamar estudio de caso la metodología ApS, investigación-acción, une el aprendizaje con el compromiso social. Optimizar la calidad del aprendizaje académico de nuestros alumnos, a la par que contribuir a la formación integral como personas socialmente responsables son las finalidades que persiguen la metodología activa ApS.

La propuesta que presentamos consiste en exponer experiencias de éxito en la metodología ApS en la Universidad de Zaragoza y mostrar las posibilidades de generar sinergias tanto a nivel interno como externo. Planteamos la posibilidad de en un futuro implicar a las distintas titulaciones del Campus de Teruel -Magisterio, Enfermería, Bellas Artes, Psicología, Administración y dirección de empresas, Ingeniería informática, Ingeniería electrónica y automática-, a través de diferentes asignaturas, de tal forma que se establezca un proceso acumulativo de experiencias que permitan tejer redes estables de colaboración y confianza entre el Campus de Teruel y el Tercer Sector turolense como forma de involucrar a la sociedad en su conjunto.

Saz, Isabel; Ramo, R.M. (2015). Aproximación a los impactos y beneficios del aprendizaje servicio en la Universidad de Zaragoza. RIDAS, Revista Iberoamericana de Aprendizaje y Servicio, 1, 9-27. DOI10.1344/RIDAS2015.1.2 


\section{La innovación social desde la universidad}

La innovación social destaca por su vinculación al campo de las mejoras sociales y los cambios que pueden operar en este sentido, se trata de un concepto dinámico, pautado por la acción social en donde el rol fundamental lo tienen los distintos agentes sociales o stakeholders (FIB, 2009). Por su parte, Murray et al. (2010), definen la innovación social como las nuevas ideas (productos, servicios y modelos) que al mismo tiempo pueden satisfacer las necesidades sociales y crear nuevas relaciones sociales o colaboraciones. Es decir, se trata de innovaciones que mejoran la capacidad de la sociedad para actuar. De acuerdo al análisis de Villa y López (2012), suele encontrarse entre las definiciones de innovación social algunos aspectos comunes:

- La innovación social debe ser gestionada por los actores facilitadores, quienes pueden revestir características diferentes. Pueden ser instituciones públicas, organizaciones no lucrativas, o bien emprendedores sociales.

- La innovación debe resolver un problema social. La innovación se presenta como una iniciativa que intenta dar una respuesta de forma novedosa a un problema o situación social.

- La solución propuesta por este tipo de innovación debe presentar determinadas características esenciales: eficaz, que se logren los objetivos pretendidos; eficiente, que lo haga con los mínimos recursos; sostenible, que se mantenga en el tiempo y que sea respetuosa con el medio ambiente; y, justa y equitativa, que promueva una distribución basada en la justicia social.

- La innovación social debe añadir valor y dirigirse a los intereses de la sociedad en su conjunto.

- La innovación social es un proceso, no es algo puntual, sino un recorrido por distintas etapas de germinación de ideas, materialización, primeros resultados, institucionalización y, en última instancia, de transformación social.

Así la Unión Europea en la Estrategia 2020 define la innovación social como otra forma de responder a las nuevas realidades y los desafíos actuales que se plantean:

(...) la innovación social puede ser un paso adelante en la forma de hacer frente a los desafíos sociales y la crisis que la UE afronta. En un momento de restricciones presupuestarias, la innovación social responde de forma efectiva a los desafíos sociales, movilizando la creatividad de las personas para desarrollar soluciones y utilizar los escasos recursos de una manera más adecuada (European Commission, 2011, 7).

La Comisión Europea clasifica las innovaciones sociales atendiendo a tres enfoques (European Commission, 2011): a) La perspectiva de la demanda social considera la

Saz, Isabel; Ramo, R.M. (2015). Aproximación a los impactos y beneficios del aprendizaje servicio en la Universidad de Zaragoza. RIDAS, Revista Iberoamericana de Aprendizaje y Servicio, 1, 9-27. DOI10.1344/RIDAS2015.1.2 
innovación como respuesta a grupos y comunidades vulnerables y con menor capacidad de implicarse y beneficiarse de la economía de mercado. b) La perspectiva de los desafíos sociales sugiere que la innovación se caracteriza por abordar desafíos sociales a través de nuevas relaciones entre actores sociales. c) La perspectiva de los cambios sistémicos plantea que la innovación hace referencia a un proceso generador de cambios sostenidos en las actitudes y el comportamiento de los actores sociales y las relaciones establecidas entre ellos.

Como se observa en el caso de la Universidad de Zaragoza, las universidades tienen como base fundamental en su misión el servicio a la comunidad. La responsabilidad y el compromiso hacia el servicio a la comunidad ha sido un pilar básico en la creación de muchas universidades europeas. Las instituciones de educación superior, a través de sus funciones de docencia, investigación y extensión, desarrolladas en contextos de autonomía institucional y libertad académica, deberían incrementar su mirada interdisciplinaria y promover el pensamiento crítico y la ciudadanía activa, lo cual contribuye al logro del desarrollo sustentable, la paz, el bienestar y el desarrollo, y los derechos humanos, incluyendo la equidad de género (UNESCO, 2009).

El aprendizaje servicio puede ser asociado a la innovación social en lo que se refiere a su desarrollo e implementación dentro de la universidad de Zaragoza, y desde el año 2009 se vienen desarrollando proyectos de innovación docente ligados a la metodología de ApS en diferentes titulaciones. Esta metodología proporciona significado y aplicación al aprendizaje al permitir desarrollar diferentes contenidos curriculares, facilitando la práctica de la planificación, el esfuerzo y el compromiso. El ApS entendido como un tipo de práctica comunitaria permite activar el ejercicio de numerosas competencias del curriculum, contemplando la iniciativa personal y la competencia social y ciudadana (Arranz, 2011). Con el objetivo de compartir recursos y experiencias, todos los proyectos en esta línea de trabajo se agrupan en torno a un mismo marco de actuación, el proyecto de ApS en la Universidad de Zaragozai.

\section{Caracterización de la metodología de Aprendizaje Servicio}

De acuerdo con Martínez-Odría (2007), hay una serie de componentes que se consideran configuradores del aprendizaje servicio y que lo distinguen de otros conceptos afines como son el voluntariado o las experiencias de aprendizaje basado en la comunidad. La autora señala los siguientes: protagonismo de la voz del alumno, este es considerado un aspecto clave de los programas; atención a una necesidad real que surge desde la comunidad; conexión con los objetivos curriculares de aprendizaje; ejecución de un proyecto de servicio; reflexión). La metodología de aprendizaje ha supuesto un cambio significativo en las dinámicas de los contextos educativos y en su

Saz, Isabel; Ramo, R.M. (2015). Aproximación a los impactos y beneficios del aprendizaje servicio en la Universidad de Zaragoza. RIDAS, Revista Iberoamericana de Aprendizaje y Servicio, 1, 9-27. DOI10.1344/RIDAS2015.1.2 


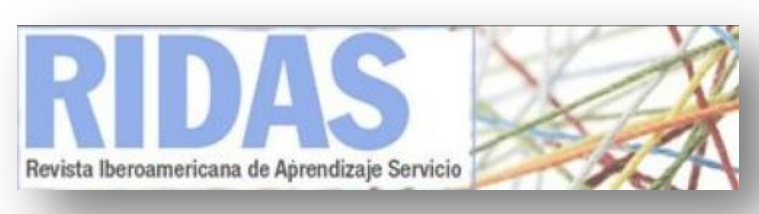

relación con el entorno social y comunitario. De acuerdo con Puig (2009) las características fundamentales que definen el aprendizaje servicio son:

- Se trata de una metodología educativa utilizable en la educación formal y no formal, aplicable con diferentes poblaciones y en diferentes contextos.

- Propone un servicio a la comunidad que permita el aprendizaje y la colaboración en un marco de reciprocidad entre el contexto comunitario y los servicios educativos.

- Se basa en la adquisición por el alumno de los contenidos clásicos de la enseñanza, junto a la adquisición de conocimientos y competencias transversales.

- Se basa en la reflexión sobre el entorno social, sobre sus dinámicas de funcionamiento y sobre los problemas y desigualdades sociales.

- Requiere de la colaboración entre instituciones educativas y entidades sociales de servicios a la comunidad.

- Provoca cambios en diferentes niveles, tanto individuales, grupales, institucionales o comunitarios, así como de las políticas sociales y educativas vigentes.

- Para su correcta implementación requiere de la implicación de los diferentes grupos de interés afectados.

Por su parte Kolenko et al. (1996) indican que el aprendizaje servicio se basa en el ciclo de aprendizaje experiencial en cuatro etapas de Kolb (1984) para entender el poder del aprendizaje servicio: a) experiencia concreta, b) observación reflexiva, c) conceptualización abstracta, d) aplicación a nuevas experiencias. El aprendizaje experiencial se entiende como un proceso en el que el conocimiento es creado a través de la transformación de la experiencia (Kolb 1984 en Kolenko et at., 1996). El contacto con la comunidad es la base de nuevas explicaciones, justificaciones y comportamientos que permite construir nuevos conceptos de la realidad y ajustar los conceptos personales ineficaces. Es aquí donde surge el potencial real de aprendizaje personal.

Las experiencias de aprendizaje servicio para tener éxito deben contener cuatro elementos, las cuatro R: realidad, reflexión, reciprocidad y responsabilidad (Godfrey et al. 2005). Kolenko et al (1996) identifican tres componentes en los proyectos de aprendizaje servicio: visión personal, comprensión de cuestiones sociales y aplicación de capacidades. A través del aprendizaje servicio, los estudiantes trabajan con y aprenden de organizaciones de la comunidad con quienes establecen alianzas para un cambio positivo del mundo real (Kenworthy-U'Ren 2008).

Saz, Isabel; Ramo, R.M. (2015). Aproximación a los impactos y beneficios del aprendizaje servicio en la Universidad de Zaragoza. RIDAS, Revista Iberoamericana de Aprendizaje y Servicio, 1, 9-27. DOI10.1344/RIDAS2015.1.2 


\section{El aprendizaje servicio en el Campus de Teruel}

En las últimas décadas se ha consolidado el Campus de Teruel, descentralización de la Universidad de Zaragoza. Este Campus agrupa a la Facultad de Ciencias Sociales y Humanas donde se imparten las titulaciones de ADE, Psicología, Bellas Artes, Grado Magisterio en Educación Infantil y Magisterio en Educación Primaria, la Escuela Universitaria Politécnica, la Escuela Universitaria de Enfermería (centro adscrito a la Universidad de Zaragoza), el Colegio Mayor Universitario Pablo Serrano, la Universidad de Verano de Teruel con una trayectoria y amplia oferta académica para la época estival, y la Fundación "Antonio Gargallo" que focaliza su actividad esencialmente en la potenciación de la investigación.

El nuevo papel de la universidad y por ende de nuestro Campus encaja plenamente con lo que las principales instituciones económicas reclaman al respecto. Es el caso de la OCDE cuando señala que "las instituciones de educación superior deben hacer algo más que solamente formar e investigar [...] han de comprometerse con otras en sus regiones, ofrecer oportunidades para un aprendizaje a lo largo de la vida y contribuir al desarrollo de puestos de trabajo intensivos en conocimiento" (Unceta 2007).

Existen muchos trabajos que han subrayado diferentes aspectos sobre el rol de la universidad en la sociedad analizando estos estudios nuestra institución y nuestro centro, la Facultad de Ciencias Sociales y Humanas se identifica con los mismos, entre los que podemos señalar:

- Un momento de replanteamiento de sus métodos de trabajo, de cara a mejorar la contribución al desarrollo.

- Participación en alianzas para el impulso de la innovación, transferencia, de métodos de trabajo efectivos y eficientes, así como la mejora al acceso de servicios para aquella población más desfavorecida partiendo de objetivos comunes.

- La universidad como espacio orientado a la investigación, a la creación y transmisión de saberes.

- La universidad como institución orientada a la formación integral de las personas, tanto en el ámbito profesional como más general.

- La universidad como espacio para la crítica y el debate, para el contraste de ideas, para la asunción de la diversidad

- La universidad como institución comprometida con su entorno, con los problemas de la sociedad en la que vive

- La universidad como ámbito para la defensa de valores universales, como espacio para la educación en la ciudadanía global o cosmopolita en el que

Saz, Isabel; Ramo, R.M. (2015). Aproximación a los impactos y beneficios del aprendizaje servicio en la Universidad de Zaragoza. RIDAS, Revista Iberoamericana de Aprendizaje y Servicio, 1, 9-27. DOI10.1344/RIDAS2015.1.2 


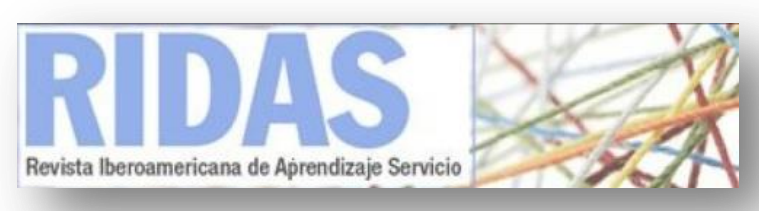

toman cuerpo asunto tales como la defensa de la Paz y los Derechos Humanos, la Sostenibilidad o la Cooperación al Desarrollo.

El Real Decreto 1791/2010, de 30 de diciembre de 2010, por el que se aprueba el Estatuto del Estudiante Universitario habla sobre que son "las Universidades (....) las que favorecerán las prácticas de responsabilidad social y ciudadana que combinen aprendizajes académicos en las diferentes titulaciones con prestación de servicio en la comunidad orientado a la mejora de la calidad de vida y la inclusión social (...) el escenario que dibuja el Espacio Europeo de Educación Superior reclama una nueva figura del estudiante como sujeto activo de su proceso de formación, con una valoración del trabajo dentro y fuera del aula, y el apoyo de la actividad docente y sistemas tutoriales". Esta metodología del saber hacer "aprendiendo haciendo", está teniendo diferentes impactos no solo en la forma de entender la combinación teoría práctica, sino en la forma de plantear desde diferentes instituciones programas y proyectos donde la unión de los distintos sectores aplican su propia estrategia de implementación, adaptando la realidad de las diferentes instituciones a la formación académica y viceversa, por lo que supone una innovación educativa y una innovación en el plano social, un poner en valor buenas prácticas que ya se vienen desarrollando, que no vienen definidas como ApS, o bien no se han desarrollado a fondo las posibilidades del vínculo entre el aprendizaje curricular y el servicio a la comunidad.

La puesta en marcha en la Facultad de Ciencias Sociales y Humanas de Teruel es reciente, tan solo hace un año que algunos profesores se incorporaron al proyecto ApS de la Universidad de Zaragoza, dedicado a desarrollar y divulgar prácticas de ApS en y desde nuestra institución. Para ello se han propuesto procesos de enseñanzaaprendizaje en los que estuvieran presentes experiencias que conllevaran simultáneamente un aprendizaje y un servicio; un aprendizaje para el estudiante y un servicio a la comunidad. La dedicación de los estudiantes y de las instituciones ha supuesto una complementariedad y aprendizaje bidireccional, ya que en todo momento se tiene claro el papel de cada uno y lo que se espera de las partes, se ha planificado pensando en cómo aprovechar el máximo tiempo y los conocimientos de las partes, así como sus posibilidades para hacer sostenible el proyecto; se han creado grupos de trabajo de apoyo desde la universidad y desde las propias instituciones; comunicación constante. La experiencia práctica está abierta para ser implementada con cualquier institución, asociación o entidad sin ánimo de lucro.

\section{Experiencia I. Asignaturas de grado de Magisterio}

Optimizar la calidad del aprendizaje académico de nuestros alumnos, a la par que contribuir a la formación integral como personas socialmente responsables son las finalidades que persiguen la metodología activa ApS.

Saz, Isabel; Ramo, R.M. (2015). Aproximación a los impactos y beneficios del aprendizaje servicio en la Universidad de Zaragoza. RIDAS, Revista Iberoamericana de Aprendizaje y Servicio, 1, 9-27. DOI10.1344/RIDAS2015.1.2 


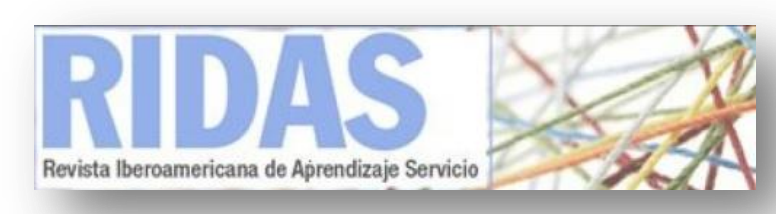

Nuestra experiencia está basada en prácticas exitosas en el campo de la Educación, llevadas a cabo en los otros dos Campus donde se imparten los grados de Magisterio de la Universidad de Zaragoza. Son variadas las experiencias que se están desarrollando, pero una mención especial es la que se referencia, ya que el trabajo continuado de un grupo nutrido de profesores de diferentes áreas durante 12 años han hecho involucrar a una ciudad entera en un proyecto común con un objetivo común: "La ciudad de las niñas y de los niños"ii en Huesca. Esta experiencia inicia su andadura en el año 2011 generando un proyecto en el que se involucra a la ciudad oscense, desarrollando los tres objetivos que le permitirán la puesta en marcha social y ciudadana del proyecto: 19) el Ayuntamiento de Huesca firma su adhesión a la red de Ciudades de niñas y niños, el 21 de octubre de 2011, en presencia de Francesco Tonucci; 20) Constitución del Consejo de las niñas y los niños, en presencia de toda la ciudad, el 6 de marzo de 2012; 3) Constitución del Laboratorio de La ciudad de las niñas y los niños de Huesca, el 26 de abril de 2012. La experiencia toma al niño como parámetro, y persigue su participación social, se trata de un proyecto para la ciudad y la ciudadanía. Así, en el proyecto no sólo participan los niños, también todas las personas interesadas en mejorar la ciudad y hacerla cada vez más habitable y humanizada: el Ayuntamiento con los técnicos y profesionales implicados en el desarrollo de la ciudad, los padres y madres, colegios, institutos y universidad, asociaciones de vecinos $y$ de comerciantes, asociaciones ciudadanas $y$, en definitiva, toda la ciudadanía.

La experiencia que durante este año ha desarrollado la Facultad de Ciencias Sociales y Humanas, en el Campus de Teruel, no es tan ambiciosa, pero sí inicia sus primeros pasos en una población rural en el que el Centro de Educación Especial es una referente para toda la Comunidad no solo local si no comarcal.

Los objetivos de la actividad han sido: 1) Conocer y aplicar los principios del diseño, la planificación y la evaluación en Educación Especial conectando la teoría con la realidad a través de su implementación. 2) Diseñar, planificar y evaluar procesos de enseñanzaaprendizaje, tanto individualmente como en colaboración con otros teniendo en cuenta el contexto, los contenidos, la metodología y la evaluación. 3) Crear una red de trabajo y colaboración entre la universidad y el Centro de Educación Especial, otros centros educativos e instituciones y 4) Contribuir en la formación integral del alumnado.

Desde el Grado de Magisterio en Educación Infantil los estudiantes de 3으 han aprendido a trabajar en necesidades reales del entorno con la finalidad de mejorarlo. El apoyo a entidades sociales, colectivas, es uno de los proyectos de aprendizajeservicio más desarrollado.

Saz, Isabel; Ramo, R.M. (2015). Aproximación a los impactos y beneficios del aprendizaje servicio en la Universidad de Zaragoza. RIDAS, Revista Iberoamericana de Aprendizaje y Servicio, 1, 9-27. DOI10.1344/RIDAS2015.1.2 


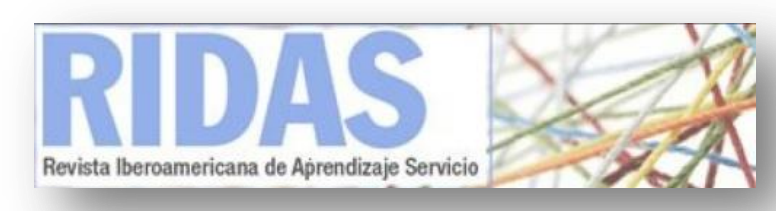

Los participantes han sido los propios alumnos tanto del grupo de la mañana como de la tarde en la asignatura "La Escuela de Educación Infantil" y "Educación Física en Educación Infantil". El proyecto inicia cuando desde el Centro de Educación Especial Gloria Fuertes de Andorra, Teruel nos solicitan una visita a la Universidad para realizar una actividad dentro de la titulación de Bellas Artes. Es a partir de este momento cuando se inicia un trabajo conjunto e interdisciplinar no solo con el C.E.E. sino también con docentes y profesores de diferentes grados y asignaturas, en concreto Bellas Artes y Grado Magisterio en Educación Infantil y Grado Magisterio en Educación Primaria contribuyendo desde nuestras asignaturas a través de un proyecto a la elaboración de una actividad no sólo de modelado, desarrollo actividad física y relaciones sociales. A partir de la experiencia se elabora una propuesta de trabajo en el que la finalidad era la convivencia entre estudiantes de diferentes edades con estudiantes de Magisterio. El tiempo de trabajo en aula fue de 30 horas y el tiempo de trabajo fuera del aula fue de 10 horas, entendiendo fuera como la práctica desarrollada en el centro educativo en dos jornadas lectivas de 5 horas cada una.

El compromiso por las partes se ha dado en las dos direcciones dándose unas condiciones favorables para ese proceso de trabajo y crecimiento conjunto que ha generado cambios. Una clara visión de las metas que se pretenden conseguir, los resultados a lo largo del proceso, la secuenciación, la concienciación y aprendizaje para el estudiante y un servicio a la comunidad han hecho del proyecto una adaptación a las demandas de la sociedad.

Todo proyecto cuenta con cuatro etapas bien diferenciadas las cuáles quedan reflejadas en la elaboración del proyecto:

1a etapa de diagnóstico y planificación: donde partiendo de la necesidad del centro y de forma colaborativa se planifica la acción de forma conjunta. Se establecen las bases del trabajo por ambas partes, por parte del C.E.E. plantean fortalecer las relaciones sociales de jóvenes con discapacidad con iguales donde todos aprendan de todos, se desarrolla un programa dentro del marco habilidades para la vida. Tomamos como referencia la propuesta de la OMS (1993) "La educación en Habilidades para la Vida persigue mejorar la capacidad para vivir una vida más sana y feliz, intervenir sobre los determinantes de la salud y el bienestar, y participar de manera activa en la construcción de sociedades más justas, solidarias y equitativas".

$2^{a}$ etapa de ejecución: elaboración del proyecto diseño, planificación en pequeños grupos de trabajo por parte de los alumnos con el acompañamiento del profesor y de la institución educativa. Una vez diseñados y elaborados los materiales contando con los recursos propios y los recursos externos se envía al centro receptor para su evaluación y posibles modificaciones o propuestas para su puesta en práctica.

Saz, Isabel; Ramo, R.M. (2015). Aproximación a los impactos y beneficios del aprendizaje servicio en la Universidad de Zaragoza. RIDAS, Revista Iberoamericana de Aprendizaje y Servicio, 1, 9-27. DOI10.1344/RIDAS2015.1.2 


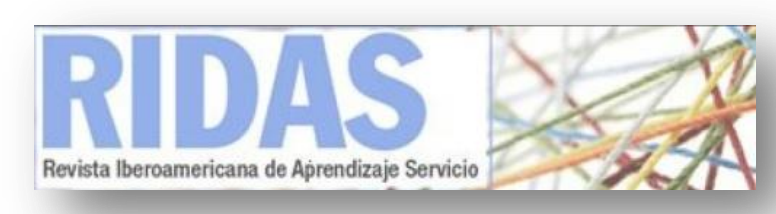

3a etapa de implementación: implementación por parte del grupo de estudiantes, gestión del proyecto ApS. Dos jornadas lectivas teniendo en cuenta el horario del Centro educativo lunes y martes.

4á etapa de evaluación y cierre: asamblea después de las sesiones, evaluación y sistematización de la actividad. Reconocimiento por parte de todos los participantes del proyecto tanto los sujetos agentes como por parte de los sujetos que reciben la acción. Evaluación del propio proceso por ambas partes. Comunicación de los resultados.

\section{Principales resultados obtenidos}

Con relación a los Objetivos 1 y 2, los alumnos han sido capaces de realizar el diseño de una planificación real para llevarla a la práctica durante dos días. Han sido capaces de realizar un trabajo individual y colaborativo en el que las aportaciones de cada uno y el trabajo de cada grupo han servido para conformar un proyecto con sentido. Han contextualizado de forma adecuada. Reconocen algunas condiciones significativas y las relacionan con los documentos de centro en concreto y con la realidad del mismo. Seleccionan y relacionan objetivos, contenidos, recursos y actividades coherentes.

Con relación al objetivo 3 , se ha creado un vínculo de trabajo conjunto con nuevas propuestas futuras. Esta acción ha llevado a crear nuevos nudos o relaciones. Y con relación al 4, el aprendizaje ha tenido sentido para los estudiantes y les ha ayudado a tener una aproximación más cercana a la realidad.

\section{Experiencia II. Asignatura del grado de Administración y Dirección de Empresas}

La asignatura en la que se va a implementar la metodología de aprendizaje servicio es "Política de Empresa". Esta asignatura es continuación de la asignatura "Dirección Estratégica I", se imparte en el tercer curso de la titulación y su peso es de 6 créditos ECTS. El objetivo general de la misma es profundizar en el estudio de la gestión empresarial desde un punto de vista globalizador, integrando los conocimientos adquiridos en otras asignaturas troncales y obligatorias. En la asignatura de "Política de Empresa" se amplía la perspectiva del análisis en el diseño de la estrategia, reconociendo expresamente el carácter dinámico de la estrategia, en línea con los desarrollos recientes, y que proceden tanto del ámbito académico como de la propia gestión empresarial. Ello exige un replanteamiento continuo de la estrategia, que deberá tener en cuenta en todo momento la actuación de los diferentes rivales, así como la evolución de un entorno en constante cambio. En concreto, el objetivo de la asignatura "Política de Empresa" es estudiar los determinantes que explican las

Saz, Isabel; Ramo, R.M. (2015). Aproximación a los impactos y beneficios del aprendizaje servicio en la Universidad de Zaragoza. RIDAS, Revista Iberoamericana de Aprendizaje y Servicio, 1, 9-27. DOI10.1344/RIDAS2015.1.2 
diferencias entre los resultados de las empresas y organizaciones en un sector determinado.

Por otro lado, a partir de un trabajo previo realizado se constata la existencia de diversos problemas en las organizaciones del Tercer Sector de Acción Social en la provincia de Teruel, agudizados por el impacto de la situación actual de reestructuración del estado de bienestar. Entre otros, los problemas identificados tienen que ver con la intensificación de la competencia en la provisión de bienes y servicios; con la falta de propuestas para la gestión de las organizaciones en un periodo de crisis coherentes con su misión, sus principios y valores; con la débil articulación territorial que repercute tanto en la incidencia social como en la política; con la inexistencia de criterios comunes o compartidos para la relación con diferentes stakeholders, como las empresas, los medios de comunicación, los sindicatos o las universidades; y con la débil relación articulada con la sociedad civil.

Hay que señalar que la experiencia educativa que proporciona el aprendizaje servicio consigue combinar el rigor académico con la relevancia de la práctica y contribuye a solucionar algunas deficiencias de la educación empresarial. La educación en el ámbito de la empresa está centrada en un conocimiento funcional en lugar de cros-funcional y holístico, orientada a proporcionar unas herramientas prácticas de resolución de problemas en lugar de un profundo conocimiento teórico, con una visión de la humanidad y de las interacciones humanas en términos puramente transaccionales, y una moralidad que reconoce la supremacía de la riqueza de los accionistas (Godfrey et al., 2005). Kolenko et al. (1996), pioneros en el análisis y defensa del aprendizajeservicio en la educación sobre management, consideran que la responsabilidad sobre el crecimiento personal y el servicio a la comunidad es una demanda en la agenda de las habilidades a desarrollar en la educación sobre management. Esto requiere ir más allá de desarrollar la conciencia cognitiva y moral sobre cuestiones sociales. Precisa educar la responsabilidad social de los estudiantes sobre sus propias acciones o las de sus empresas. Para este fin proponen los proyectos de aprendizaje servicio como una poderosa opción. En nuestro caso proponemos el aprendizaje servicio en las organizaciones no lucrativas que prestan servicios sociales a la comunidad.

Para implementar la experiencia de aprendizaje y servicio en la asignatura "Política de Empresa" se siguen las fases establecidas por Pizarro y Jerez, (2012), adaptándolas a nuestra titulación y a las entidades con las que vamos a colaborar:

- Vinculación con organizaciones del Tercer Sector de Acción Social de la provincia de Teruel y a continuación se seleccionan aquellas entidades que muestran mayor compromiso y motivación frente a la colaboración en el proyecto conjunto.

Saz, Isabel; Ramo, R.M. (2015). Aproximación a los impactos y beneficios del aprendizaje servicio en la Universidad de Zaragoza. RIDAS, Revista Iberoamericana de Aprendizaje y Servicio, 1, 9-27. DOI10.1344/RIDAS2015.1.2 


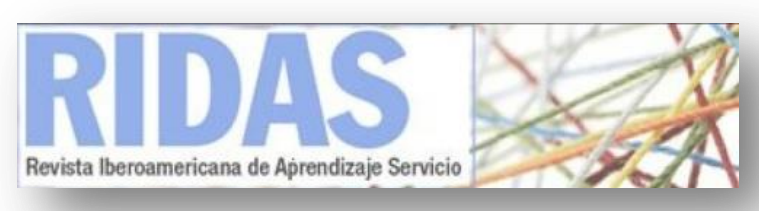

- Contextualización del proyecto de aprendizaje servicio. Esta etapa se trata de explicar al estudiante el objetivo de su participación en el proyecto.

- Formación y Gestión de equipos. El propósito de esta fase tiene como objetivo desarrollar estrategias para la asignación de tareas y la resolución de conflictos grupales.

- Establecimiento de compromisos. En este momento se procede a establecer reuniones entre la entidad y el grupo de estudiantes para establecer la cronología del trabajo a desarrollar.

- Fase de desarrollo e inmersión. En esta etapa se procede al desarrollo y consecución de los compromisos propuestos a través de las diversas actividades establecidas.

- Presentación y comunicación. Esta fase tiene como propósito comunicar los resultados del proyecto realizado por los estudiantes, con el asesoramiento y visto bueno del profesor.

- Generación de capacidades y evaluación. En esta etapa se produce un análisis por parte de las organizaciones de la propuesta efectuada por los estudiantes.

\section{Impacto y beneficios del Aprendizaje Servicio}

Las universidades deben tener en cuenta los beneficios de ser más proactivos en la adaptación de métodos innovadores de promoción y facilitación de la participación estudiantil y el voluntariado. Por otra parte, creemos que este tipo de innovación debe estar vinculada a los resultados de impacto medibles e identificables. De tal forma que la aplicación siga algunas estrategias que pueden conducir a un programa de mayor impacto:

- Adoptar un enfoque interdisciplinar en el que haya un desarrollo transversal de los conocimientos que se desarrollan

- Proporcionar oportunidades para el liderazgo para todas las partes involucradas -los estudiantes, el personal, la institución y la comunidad-

- Crear experiencias significativas que ayuden a los estudiantes adquirir experiencia en el mundo real

- Establecer reconocimiento y recompense a las partes interesadas participantes en el programa

- Lograr flexibilidad para desarrollar un compromiso socialmente sensible con las necesidades y circunstancias cambiantes de los estudiantes universitarios y la comunidad en la que se desarrolla el programa

Los trabajos que examinan el aprendizaje servicio también muestran resultados e impactos positivos. En conjunto, esos estudios sugieren que la participación en el 
aprendizaje servicio se asocia con resultados positivos para los jóvenes, con un efecto moderado sobre los resultados académicos y pequeños efectos para los resultados no cognitivos, incluyendo las habilidades sociales, auto-percepción y motivación (Morrison Gutman y Schoon, 2013). Por ejemplo, Billig (2000) encuentra que la participación en los programas de aprendizaje servicio está asociada con resultados positivos en el ámbito académico, personal, profesional y en lo que respecta a resultados cívicos. Así mismo, Melchior y Ballis (2002) encuentran que hay una reducción en el ausentismo de los participantes de la escuela secundaria.

La literatura sobre resultados de los estudiantes relacionados con la participación en el aprendizaje servicio es muy amplia y apunta a un cambio multidimensional en los estudiantes como resultado de la participación (Swaner y Brownell, 2009). Roldan et al. (2004) establecen una triple categorización de los impactos en los estudiantes que participan en el programa: a) logro académico; b) participación cívica; y c) crecimiento personal.

Por lo que respecta al logro académico, Pascarella y Terenzini (2005) en su investigación en la educación superior, encontraron que los estudiantes que participan en el servicio comunitario tenían una ventaja de aprendizajes mensurables sobre los que no están involucrados en el servicio. En la misma línea se pronuncia el estudio de Eyler y Giles (1999) que ponen de relieve las posibles razones que llevan a los estudiantes a un mayor logro académico gracias al aprendizaje servicio. Así, los estudiantes sugieren que el aprendizaje servicio les lleva a estar más comprometidos y a tener mayor curiosidad por cuestiones que experimentan en la comunidad y que se vincula con los contenidos del curso. Los estudiantes encuentran que pueden usar el material que ellos aprenden a partir del contexto de la comunidad rica y compleja. El impacto del aprendizaje servicio parece aplicarse a una serie de resultados académicos, de tal forma que la participación en el aprendizaje servicio se asocia con mayor probabilidad de obtención de un título y una mayor interacción con el profesorado, junto con avances en el conocimiento académico (Astin et al., 1999); que los estudiantes fueron más propensos a informar sobre lo que habían aprendido a aplicar y también que obtenían mejores calificaciones de los cursos (Markus et al. 1993 y mayor nivel en la escritura de redacción y ensayo (Shastri, 1999; Strange, 2000).

Por lo que respecta a la participación ciudadana, diversos estudios incluyen en esta dimensión variables cognitivas, actitudinales y conductuales (Swaner y Brownell, 2009). En este sentido el estudio de Astin et al. (1999) muestra que los estudiantes que participaron en un programa de aprendizaje servicio en la universidad eran más propensos a ser voluntarios después de la universidad, asistir a la escuela de posgrado, socializar con diversas personas con más frecuencia, ayudar a otros en dificultades, desarrollar una filosofía de vida significativa, promover la comprensión racial, 


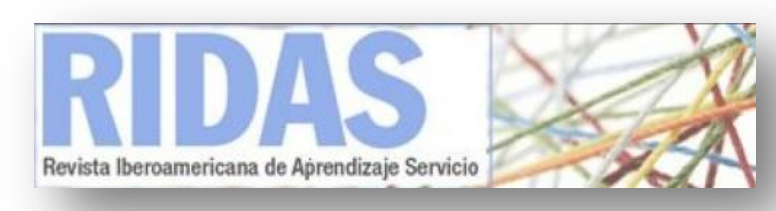

participan en la acción comunitaria y la limpieza del medio ambiente, y desarrollar la auto-eficacia. Eyler y Giles (1999) encontraron que un tercio de los participantes en el aprendizaje servicio fue capaz de desarrollar una nueva perspectiva de los problemas sociales, comportamiento cívico, y la preocupación por la responsabilidad cívica.

Por último, en el ámbito de desarrollo personal e interpersonal Eyler y Giles (1999) encontraron que la participación en un programa de aprendizaje servicio permitió a los alumnos a reducir los estereotipos y a desarrollar mayor tolerancia, también contribuyó de forma positiva en la capacidad de trabajar bien con los demás, a aumentar las habilidades de liderazgo $y$, en términos afectivos, los estudiantes informan que permite un mayor conocimiento de sí mismo, crecimiento espiritual, y la búsqueda de recompensa en ayudar a los demás. Además, el aprendizaje servicio puede incrementar la sensibilidad de los alumnos al estar más conectados a la comunidad.

Como se referencia a lo largo del trabajo la metodología práctica de aprendizaje servicio, además de logar mayor formación profesional y personal por parte de nuestros estudiantes, contribuye, aunque sea modestamente, a devolver a la sociedad la confianza que ha depositado en la universidad como organización promotora de conocimiento y de compromiso social. Así mismo, el aprendizaje servicio puede convertirse en una vía para fomentar el civismo y el voluntariado entre los jóvenes universitarios del campus de Teruel. Las instituciones de educación superior, en su papel de formadoras de profesionales, han de estar especialmente atentas a las expectativas y problemas de la sociedad para poder aportar mejoras en los programas de formación de los estudiantes (Schön, 1983). Los programas de aprendizaje servicio son una tendencia creciente a nivel mundial y la investigación sobre su impacto en la participación futura, como voluntarios, de los participantes en los programas es concluyente (Berry y Chisholm, 1999).

Esta apuesta con carácter interdisciplinar y transversal pretende involucrar a medio plazo a todas las titulaciones que se imparten en la Facultad, además de ADE, Grado de Magisterio en Educación Infantil y Grado en Magisterio en Educación Primaria, Bellas Artes, Psicología. Ya se están dando pasos para convertirse en un proyecto de Campus, ya que son varios los puntos de encuentro sinergias que se están dando con la Escuela Universitaria Politécnica de Teruel desde los propios miembros del grupo de innovación así como la demanda que se está produciendo por parte de las instituciones, organizaciones. La Facultad a través del ApS se está convirtiendo en un espacio abierto en el que todos aprenden de todos.

\section{Conclusiones}

Saz, Isabel; Ramo, R.M. (2015). Aproximación a los impactos y beneficios del aprendizaje servicio en la Universidad de Zaragoza. RIDAS, Revista Iberoamericana de Aprendizaje y Servicio, 1, 9-27. DOI10.1344/RIDAS2015.1.2 
El aprendizaje servicio no es fácil; requiere perseverancia, motivación, dedicar más tiempo y energía que las experiencias tradicionales en el aula, cambios en varios ámbitos como en el entorno de aprendizaje, y el compromiso de múltiples stakeholders: profesores, estudiantes y representantes de organizaciones de la comunidad (Kenworthy-U'Ren 2008). El primer paso es conocerse a uno mismo para alinear las necesidades de las tres partes con distintas prioridades e intereses y poder trabajar colectivamente para conseguir aprendizajes mutuos.

Desde nuestro punto de vista, la experiencia puede favorecer la generación de una comunidad de aprendizaje, conformada por un conjunto de actores entre los que se establece una dinámica de aprendizaje: la organización no lucrativa, el estudiante y el docente. La organización no lucrativa entrega su experiencia como aprendizaje en el ámbito de los servicios sociales de la comunidad. El estudiante comparte sus aprendizajes con la organización no lucrativa, por medio de la generación de soluciones creativas y, a la vez, construye su propia experiencia de aprendizaje en base al proceso de "aprender haciendo". Por último, el profesor propicia e implementa las acciones de aprendizaje pertinentes para la experiencia, adecuándolas al programa curricular de la asignatura y a los conocimientos formales que los estudiantes deben adquirir.

Se esperan ciertos resultados de la aplicación de los programas de aprendizaje servicio: a) una comprensión más profunda de los contenidos del curso, b) un conocimiento práctico del proceso de toma de decisiones, c) la adquisición de estrategias para transferir conocimiento y habilidades en la resolución de conflictos, d) considerar información y puntos de vista conflictivos, e) solucionar problemáticas y comprender que dichas soluciones son tentativas y que hay que lograr niveles más avanzados de desarrollo cognitivo (Eyler, 2000). Por otra parte, el aprendizaje servicio permite fortalecer los vínculos de la organizaciones sociales con la universidad, lo cual puede es de gran importancia y es uno de los objetivos que debe perseguir la universidad.

\section{Referencias bibliográficas}

Arranz, P. (2011). La Universidad de Zaragoza en materia de Responsabilidad Social. En I. Saz Gil (Coord.), Contribución de las organizaciones a la consecución de los objetivos de desarrollo del milenio. Valencia: Tirant lo Blanch.

Astin, A.W., Sax, L.J., \& Avalos, J. (1999). Long term effects of volunteerism during the undergraduate years. Review of Higher Education, 22, 187-202.

Saz, Isabel; Ramo, R.M. (2015). Aproximación a los impactos y beneficios del aprendizaje servicio en la Universidad de Zaragoza. RIDAS, Revista Iberoamericana de Aprendizaje y Servicio, 1, 9-27. DOI10.1344/RIDAS2015.1.2 
Batlle, R (2010). Aprendizaje-Servicio en España 2010. ASHOKA. Recuperado de http://roserbatlle.files.wordpress.com/2009/02/informe-resumido-aps-espac3b1a2010.pdf

Berry, H.A., \& Chisholm, L.A. (1999). Service-learning in higher education around the world: An initial look. New York: The International Partnership for Service-Learning.

Billig, S.H. (2000). Research on K-12 school-based service-learning. Phi Delta Kappan, 81(9), 658-664.

European Commission (2011). Empowering people, driving change: Social innovation in the European Union. Luxembourg: Publications Office of the European Union.

Eyler, J. (2000). What Do We Most Need to Know About the Impact of Service-Leaming on Student Leaming? Michigan Joumal of Community Service-Leaming, Special Issue.

Eyler, J. y Giles, D.E. Jr. (1999). Where's the learning in service-learning? San Francisco: Jossey-Bass Publishers.

Fundación de la Innovación Bankinter, FIB (2009). Innovación Social. Reinventando el desarrollo sostenible. Fundación de la Innovación Bankinter. Recuperado de http://www.fundacionbankinter.org/system/documents/8159/original/XII_FTF_Innova cion_Social_CASTELLANO.pdf

Godfrey, P.C., Illes, L.M. \& Berry, G. (2005). Creating Breadth in Business EducationThrough Service-Learning. Academy of Management Learning \& Education, 4, 309-323.

Kenworthy-U'Ren, A.L. (2008). A Decade of Service-Learning: A review of the Field Ten Years after JOBES's Seminal Special Issue. Journal of Business Ethics, 81, 811-822

Kolb, D.A. (1984). Experiential Learning. Experience as the Source of Learning and Development. New Jersey: Prentice-Hall.

Kolenko, T.A., Porter, G., Wheatley, W. \& Colby, M. (1996). A Critique of Service learning Projects in Management Education. Journal of Business Ethics, 15, 133-142.

Madrid Villa, A. \& López, A.L. (2012). La evaluación de la innovación social en las universidades: un primer acercamiento. En D. Montero, D. y P. Fernández de Larrinoa (Eds.), Calidad de vida, inclusión social y procesos de intervención (pp. 305-328). Bilbao: Publicaciones de la Universidad de Deusto.

Saz, Isabel; Ramo, R.M. (2015). Aproximación a los impactos y beneficios del aprendizaje servicio en la Universidad de Zaragoza. RIDAS, Revista Iberoamericana de Aprendizaje y Servicio, 1, 9-27. DOI10.1344/RIDAS2015.1.2 


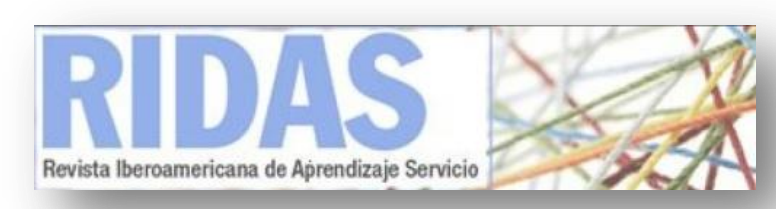

Markus, G.B., Howard, J.P.F. y King, D.C. (1993). Integrating community service and classroom instruction enhances learning: Results from an experiment. Educational Evaluation and Policy Analysis, 15(4), 410-419.

Martínez-Odría, A. (2007). Service-learning o aprendizaje-servicio. La apertura de la escuela a la comundidad local como propuesta de educación para la ciudadanía. Bordón, 59 (4), 627-640.

Melchior, A. \& Bailis, L.N. (2002). Impact of service-learning on civic attitudes and behaviors of middle and high school youth: Findings From Three National Evaluations. In A. Furco and S. Billig (Eds.), Service-Learning: the essence of the pedagogy . Vol. 1. Advances in Service-Learning Research. Greenwich, CT: Information age.

Real Decreto 1791/2010 (2010). Aprueba el Estatuto del Estudiante Universitario. Ministerio de Educación.

Recuperado de http://www.boe.es/boe/dias/2010/12/31/pdfs/BOE-A-2010-20147.pdf

Morrison Gutman L. \& Schoon, I. (2013). The impact of non-cognitive skills on outcomes for young people Literature review. Londres: Institute of Education, University of London.

Murray, R., Cavlier-Grice, J. \& Mulgan, G. (2010). The Open Book of Social Innovation. NESTA (The National Endowment for Science Technology and the Arts) and Young Foundation.

Recuperado de

http://www.nesta.org.uk/sites/default/files/the_open_book_of_social_innovation.pdf

Organización Mundial de la Salud (OMS) (1993). División Salud Mental: Iniciativa Internacional para la Educación en Habilidades para la Vida en las Escuelas. Ginebra: OMS.

Pascarella, E.T., \& Terenzini, P.T. (2005). How college affects students: A third decade of research (Volume 2). San Francisco: Jossey-Bass.

Pizarro, V. \& Jerez, O. (2012). El desafío de hacer rentable una empresa de menor tamaño: determinación de costos por medio de cursos de A+S. En Actas de la II Jornada de investigadores sobre aprendizaje-servicio. Buenos Aires: CLAYSS-Red Iberoamericana de aprendizaje-servicio.

Recuperado de http://www.clayss.org.ar/ingles/06_investigacion/jornadas/Libro_IIJornada-Investigadores-Aprendizaje-Servicio/CIII-18_Pizarro-Jerez.pdf

Saz, Isabel; Ramo, R.M. (2015). Aproximación a los impactos y beneficios del aprendizaje servicio en la Universidad de Zaragoza. RIDAS, Revista Iberoamericana de Aprendizaje y Servicio, 1, 9-27. DOI10.1344/RIDAS2015.1.2 


\section{RDAS}

Puig, J. (coord.) (2009). Aprendizaje servicio. Educación y compromiso cívico. Barcelona: Graó.

Roldan, M., Strage, A. y David, D. (2004). A framework for assessing academic service learning across disciplines. En M. Welch and S.H. Billig (Ed.), New perspectives in service-learning: Research to advance the field (pp. 39-59). Greenwich, CT: Information Age Publishing.

Schön, D. (1983). El profesional reflexivo. Cómo piensan los profesionales cuando actúan. Barcelona: Paidós.

Shastri, A. (1999). Investigating content knowledge gains in academic service-learning: A quasiexperimental study in an educational psychology course. Comunicación presentada en Annual Meeting of the American Educational Research Association, Montreal, Canadá.

Strange, A. (2000). Service-learning: Enhancing student learning outcomes in a college level lecture course. Michigan Journal of Community Service Learning, 7, 5-13.

Swaner, L.E. \& Brownell, J.E. (2009). Outcomes of High Impact Practices for Underserved Students: A Review of the Literature. Association of American Colleges and Universities (AAC\&U) Project USA.

UNESCO (2009). Conferencia Mundial sobre la Educación Superior: La nueva dinámica de la educación superior y la investigación para el cambio social y el desarrollo. París: UNESCO.

Recuperado de http://www.unesco.org/education/WCHE2009/comunicado_es.pdf

Unceta, K. (dir.) (2007). La cooperación al desarrollo en las universidades españolas. Alicante: Biblioteca Virtual Miguel de Cervantes.

\footnotetext{
${ }^{i}$ Para conocer más sobre el proyecto véase: http://www.unizar.es/aprendizaje servicio/

ii http://www.unizar.es/aprendizaje_servicio/experiencias.html
} 